\title{
The development of a standard qualification protocol for all climatic and thermostatic chambers according to the requirements of the Good Manufacturing Practices (GMP) and the standards of characterization: NF X 15-140 and NF EN 60068-3.
}

\author{
Lamkharbach Yassine $^{{ }^{*}}$, Bouamrani Mouna Latifa ${ }^{1}$, Bazi Fathallah ${ }^{1}$, Mourran Aicha $^{1}$, Yousfi Samia ${ }^{1}$ \\ ${ }^{1}$ Department of Chemistry, Laboratory of Analytical Chemistry and Physico-Chemistry of Materials (LACPCM), Faculty of Science \\ Ben Msik, Hassan II University.
}

\section{Article Info}

Received Aug 8, 2018

\section{Keyword: \\ Qualification protocol \\ Climatic chambers \\ Thermostatic chambers \\ NF X 15-140 \\ NF EN 60068-3 \\ Good Manufacturing \\ Practices}

\begin{abstract}
The qualification of climatic and thermostatic chambers is not based on a single standard, but it also requires the control of several standards such as NF X 15-140, NF EN 60068-3 and the requirements of the Good Manufacturing Practices (GMP). So, currently the qualifiers only take into account the standard NF X 15-140 or NF EN 60068-3 to make their qualification, without giving importance to other criteria that must be treated or accurately tested and that are not mentioned in these standards, but they are cited in other references such as GMP (Good Manufacturing Practices). Because with the standards NF X 15-140 or NF EN 60068-3 we can only judge on the conformity of the distribution of temperature and humidity into the chamber. That is to say, in qualification terms, it is the last step of the qualification, which is the PQ (Performance Qualification). While in the GMP, we find the other steps of qualification, which are the IQ (Installation Qualification) and the OQ (Operational Qualification) and even in the GMP, they are not well detailed. Thanks to the use of the characterization standards such as NF X 15140 and NF EN 60068-3 and the requirements of the Good Manufacturing Practices, we have been able to develop a standard qualification protocol. Indeed, this protocol could be used for all climatic and thermostatic chambers for the purpose of their qualification. Also, it could allow us to make a judgment on the conformity or not of the chamber to qualify.
\end{abstract}

\section{Corresponding Author:}

Lamkharbach Yassine,

Department of Chemistry, Laboratory of Analytical Chemistry and Physico-Chemistry of Materials (LACPCM),

Faculty of Sciences Ben M'sik, Hassan II University,

Av Driss El HartiSidiOthmane Casablanca B.P 7955, Maroc

Email: lamkharbachyassine@gmail.com

\section{Introduction}

TheToday's equipment is the main link between the raw materials and the finished product, so it must be installed, operational and must be maintained in accordance with its design specifications. Whatever the sector of activity, the same principles apply to all equipment. Many manufacturers and laboratories must comply with requirements concerning the control of temperature and humidity in their manufacturing processes or measurement to harmonize practices concerning the characterization and verification of chambers. The qualification of climatic and thermostatic chambers is not based on a single standard, but it also requires the control of several standards such as NF X 15-140, NF EN 60068-3 and the requirements of the Good 
Manufacturing Practices (GMP). So, currently the qualifiers only take into account the standard NF X 15-140 or NF EN 60068-3 to make their qualification, without giving importance to other criteria that must be treated or accurately tested and that are not mentioned in these standards, but they are cited in other references such as GMP (Good Manufacturing Practices). Because with the standards NF X 15-140 or NF EN 60068-3 we can only judge on the conformity of the distribution of temperature and humidity into the chamber. That is to say, in qualification terms, it is the last step of the qualification, which is the PQ (Performance Qualification). While in the GMP, we find the other steps of qualification, which are the IQ (Installation Qualification) and the OQ (Operational Qualification) and even in the GMP, they are not well detailed. Thanks to the use of the characterization standards such as NF X 15-140 and NF EN 60068-3 and the requirements of the Good Manufacturing Practices, we have been able to develop a standard qualification protocol. Indeed, this protocol could be used for all climatic and thermostatic chambers for the purpose of their qualification. Also, it could allow us to make a judgment on the conformity or not of the chamber to qualify.

\section{General information on the qualification chambers:}

Any qualification process is based on four main bases:

\section{2-1 Design Qualification:}

The design qualification is done at the time of receipt of this chamber within the industry, in this qualification stage, it is necessary to check the conformity of the order, established according to the specification on which the customer and the supplier had previously agreed. For example, all components, spare parts, technical documentation and the condition of the internal and external packaging of the equipment must be noted in the approval document, then we declare the conformity of the equipment according to order then we move to the qualification of installation.

During this qualification stage:

- The associated measuring instruments are coded (internally), listed and classified as critical or noncritical.

PS: Critical instruments are defined as instruments that have an impact on the quality or effectiveness of the finished product or those used to obtain registration data for regulatory submissions.

- Verification, calibration, maintenance, cleaning and operational procedures are identified and written.

- The verifications and calibrations are carried out before the operational qualification.

\section{2-2 Installation Qualification:}

The installation qualification corresponds to the documented verification that the equipment is installed or modified according to the specifications. It makes it possible to realize and document the installation of the equipment in the location chosen by the customer. All the documents and plans relating to the installation (drawings, material certificates, calibration certificates, certificates of conformity, description, principle, operating manual, nomenclature, electrical diagrams, etc.) must be provided. The verification of the conformity of installation is realized before any startup of a device by execution of the protocol of qualification installation.

The main installation qualification tests for climatic and thermostatic chambers:

- Check the presence of supplier plans and drawings.

- Check the presence of user manuals, maintenance (suppliers).

- Check that the equipment information match vendor documents, such as the name of the manufacturer, model, serial number for an item of equipment.

- Check that the technical environment (safety, environmental conditions, installation site ...) is in agreement with the system or equipment to be installed.

- Check that the connections to the utilities (electrical current, water, steam, compressed air, gas, vacuum ...) are in accordance with the recommendations of the manufacturer.

- Check compliance of construction capacities and materials with supplier documents and specifications.

- Check for materials certificates.

- Check for spare parts list. 
- Check that the associated measuring instruments and automate are verified and calibrated in a documented manner.

- Check the presence of all information relating to the safety of equipment and operators, training, modifications.

\section{2-3 Operational Qualification:}

The operational qualification consists of a documented verification that the various elements of the installed or modified equipment are functioning correctly under the predicted conditions. It is intended to test the equipment in the customer environment to ensure it meets the specifications previously defined [4]. Once the installation qualification has been completed, the operational qualification consists in carrying out functional tests.

All functions identified as critical will require explicit tests that consist of:

- Perform operational tests in real and empty*.

- Carry out trials that include the upper and lower operating limits.

- Perform empty safety tests.

- Perform tests to check the effectiveness of associated equipment such as alarms, recording systems, controllers ...

- Carry out simulation tests: start-up and shutdown systems.

* Some tests require the presence of the product. In this case, use a placebo.

During this qualification stage:

- You have to finish the procedures of operating, cleaning and maintenance.

- Training is needed for production and maintenance personnel.

- The system / equipment to be qualified and the associated measuring instruments are integrated into the annual qualification plans and the preventive maintenance plans.

\section{2-4 Performance Qualification:}

Once the installation and operational qualification have been approved, the performance qualification is then carried out under actual operating conditions and with the product or a placebo (the characteristics of which are close to those of the product). It consists of:

- Perform actual functioning tests with the product (or placebo) and with the user personnel.

- Check the influence of the operating parameters on the load.

- Perform functional tests on the product at each critical stage of the process.

- Perform system performance measurements.

- Ensure the reproducibility of the performances obtained in load on several tests according to the specifications and specifications pre-established.

Concerning the climatic and thermostatic chambers: The purpose of the verification is to compare the results obtained during the characterization with specifications of the chamber and to conclude the Compliant (C) or Not Compliant (NC) of the chamber for each of the specifications.

The specifications or requirements may include the following parameters:

2-4-1 Homogeneity: The characterization of the homogeneity of the temperature in the working space makes it possible to determine during the duration of the measurements the homogeneity of the temperature values given by the sensors.

2-4-2 Stability: The characterization of the stability of the temperature in the working space makes it possible to determine the maximum temperature variation at a measurement point of the working space during the measurement period.

2-4-3 Setpoint deviation: This is the difference between the Setpoint value and the air temperature or it is the Setpoint deviation which makes it possible to know the correction to be made to the Setpoint value when using the chamber to obtain the desired or otherwise specified condition. 
2-4-4 Indication error: This is the difference between the value of the indicator and the air temperature, the indication error makes it possible to characterize the representativeness of the values displayed by the environmental indicator.

2-4-5 Recovery time: The recovery time is the time required for the temperature in the chamber to return to the established regime after a disturbance.

\section{Experimental part: qualification of the thermostatic chamber.}

\section{3-1 Installation qualification:}

The purpose of this qualification is to demonstrate that the equipment meets the specification of the specifications and that its installation is carried out in compliance with the manufacturer and the requirements GMP. The tests carried out in the installation qualification:

3-1-1 Machine support: Check the final machine support. For this reason it is necessary to :

- Check that the machine is in the final and intended place for its use.

- Check the stability and the well fixed of the material on a horizontal ground.

- Check that there is enough space to allow the equipment to function properly and to facilitate access for technicians.

3-1-2 Material certificates: check that the materials of all the elements in contact with the product meet the requirements GMP.

- Check the presence and the conformity of the certificates of materials.

3-1-3 Lay out: Check the dimensions of the chamber.

Measure the dimensions of the machine using a measuring meter: Length; Width ; Height.

Example:

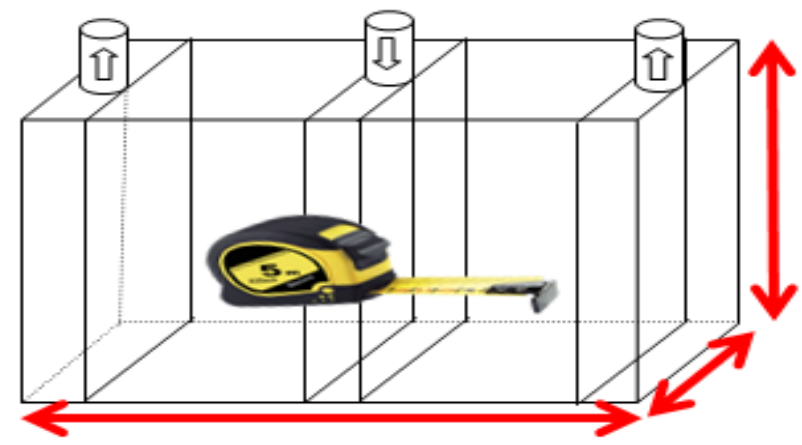

Figure 1: Example of the dimensions of a chamber.

3-1-4 Utility: Check that the power supply of the machine in utilities complies with the manufacturer's recommendations by:

- Measuring the voltage at the input, using a multimeter.

- Checking the frequency and type of power supply.

Example:

Table 1: Utility data.

\begin{tabular}{|c|c|}
\hline \multicolumn{2}{|c|}{ Electricity supply } \\
\hline \multicolumn{2}{|c|}{ Three-phase ALTernating } \\
\hline Tension & Frequency \\
\hline $380 \pm 10 \mathrm{~V}$ & $50 \mathrm{~Hz}$ \\
\hline
\end{tabular}


3-1-5 Static safety: Check the presence of the static safety elements on the chamber such as :

- Neutral connectivity.

- Grounding connectivity.

- Control elements.

3-1-6 Components: Check the presence and conformity of components: electrical, mechanical, hydraulic systems of the machine and of the Air central treatment.

\section{3-2 Operational Qualification (OQ):}

The purpose of this qualification is to demonstrate that the machine is working properly in a vacuum, in accordance with the specification of the specifications and in compliance with the requirements of the GMP. It consists in verifying, by carrying out a series of isolated dynamic tests, that all the subsystems of the machine are functional. The tests performed in the operational qualification are:

3-2-1 Control elements: In both automatic and manual modes, the following elements must be checked as: general interceptor; on / off switches; Temperature and humidity control buttons; the clock...

3-2-2 Emergency pushbutton: Demonstrate that the emergency pushbutton of the machine is functional, to do this it is only necessary to turn on the chamber and then press the button "Emergency pushbutton".the air handling unit (Blowing, Extraction, recovery) must stop, as an expected result.

3-2-3 Temperature setpoint: Demonstrate that the temperature control of the machine is functional. To do this, the chamber must be switched on and then set the product temperature setpoint of the "heating temperature controller" at $60^{\circ} \mathrm{C}$ and wait for Stabilization of the temperature at $60^{\circ} \mathrm{C}$. The expected product temperature must reach $60^{\circ} \mathrm{C}$ and the steam valve opens and closes automatically to ensure the stability of the temperature at $60^{\circ} \mathrm{C}$.

3-2-4 Safety thermostat: Check that the safety thermostat of the chamber is functional, for this you only need to set the thermostat value at $30^{\circ} \mathrm{C}$ and set the setpoint temperature value at $45^{\circ} \mathrm{C}$ then set the chamber and wait until the temperature of the product reaches $30^{\circ} \mathrm{C}$. The chamber must stop and an audible alarm will start and a "DEFAULT" LED will light up, as expected results.

3-2-5 Temperature alarm: Check that the temperature alarm of the chamber is functional. To do this, set the alarm setpoint at $45^{\circ} \mathrm{C}$ and set the product temperature setpoint of the "heating temperature controller" at $46^{\circ} \mathrm{C}$. When the alarm set point increases until it is above the product temperature, eg $47^{\circ} \mathrm{C}$, The chamber must stop and an audible alarm will sound and a "High Temperature" indicator light will be on.

3-2-6 Safety of the doors: To demonstrate that the safety of the oven doors is functional, simply turn on the chamber and open its doors. The chambre must stop, as an expected result.

3-2-7 Air flow: Use an anemometer to Measure at the inlet of the machine the air flow of the blowing, fresh air, extractor and recoveries.

3-2-8 Production test (empty): you must demonstrate that the machine is operational. For this it is necessary to:

- Set the setpoint temperature for the "controller" at $40^{\circ} \mathrm{C}$.

- Make a half-hour test.

- Set the heating time of the "timer" at $30 \mathrm{~min}$.

- On the graph, check the time, humidity, temperature on the recording.

Look at the following example:

Table 2: Parameter data of the production test.

\begin{tabular}{|c|c|c|c|}
\cline { 2 - 4 } \multicolumn{1}{c|}{} & Obtained & Standard & $(\mathrm{C} / \mathrm{NC})$ \\
\hline Temperature cycle. & 40,75 & $40 \pm 5^{\circ} \mathrm{C}$ & $\mathrm{C}$ \\
\hline Time cycle. & $30 \min 22 \mathrm{~s}$ & $30 \min \pm 5 \%$ & $\mathrm{C}$ \\
\hline Relative humidity. & $1,35 \%$ & $1,3 \pm 5 \%$ & $\mathrm{C}$ \\
\hline
\end{tabular}


3-2-9 Checking the pressure drop of the filters: Putting in place standards for filter cloggin. For this it is necessary to :

- Adjust the air flow according to the operating procedures of the chamber.

- Start an operating cycle of the chamber.

- Measure pressure drop of the air treatment filters.

- The measured pressures are initial pressures.

- $\quad$ Alert limit $=$ initial pressure $\pm 50 \%$

- $\quad$ Limit of action = maximum limits recommended by the filter manufacturer.

Look at the following example:

Table 3: The pressure drop measurements of the filters.

\begin{tabular}{|c|c|c|c|c|c|}
\hline Filters & Initial pressure & Alert limit & Limit of action & Result & C / NC \\
\hline G4 & $10 \mathrm{~Pa}$ & 5 to $15 \mathrm{~Pa}$ & $250 \mathrm{~Pa}$ & $10 \mathrm{~Pa}$ & $\mathrm{C}$ \\
\hline F9 & $27 \mathrm{~Pa}$ & 13,5 to $40,5 \mathrm{~Pa}$ & $250 \mathrm{~Pa}$ & $27 \mathrm{~Pa}$ & $\mathrm{C}$ \\
\hline H13 & $90 \mathrm{~Pa}$ & 45 to $135 \mathrm{~Pa}$ & $600 \mathrm{~Pa}$ & $90 \mathrm{~Pa}$ & $\mathrm{C}$ \\
\hline
\end{tabular}

3-2-10 Calibration: Demonstrate that all critical measuring instruments of the machine are calibrated and labeled. Then indicate the calibration certificate number, the calibration date and the recalibration date. Check the cycle registration. Check the operation of the recorder to do this:

- Run a 30 minutes production test.

- The results will be recorded in the recorder.

- Print the registration report

- Check the legibility of the data, the accuracy of the records and the curves.

\section{3-3 Performance Qualification (PQ):}

The purpose of this characterization is to know and / or periodically ensure the real performance of the chamber. It makes it possible to determine the characteristics which are the subject of specifications.

PS: In this article we have only conducted the characterization at temperature $40^{\circ} \mathrm{C}$ because the same steps apply at $50^{\circ} \mathrm{C}$ and $60^{\circ} \mathrm{C}$.

\section{3-4-1 Verification of the homogeneity of the temperature at $40^{\circ} \mathrm{C}$. "Empty chamber":}

According to the standard NF X 15-140: A volume greater than $2 \mathrm{~m} 3$ and less than or equal to $20 \mathrm{~m}^{3}$ the characterization is carried out with 15 temperature probes.

- Nine temperature probes placed as previously;

- Six temperature probes distributed at the center of the surfaces that delimit the workspace.

- Measurements are made at the minimum operating temperature: $40^{\circ} \mathrm{C}$.

So place 15 temperature probes in the chamber, as shown in the figure below “

Here is an example:

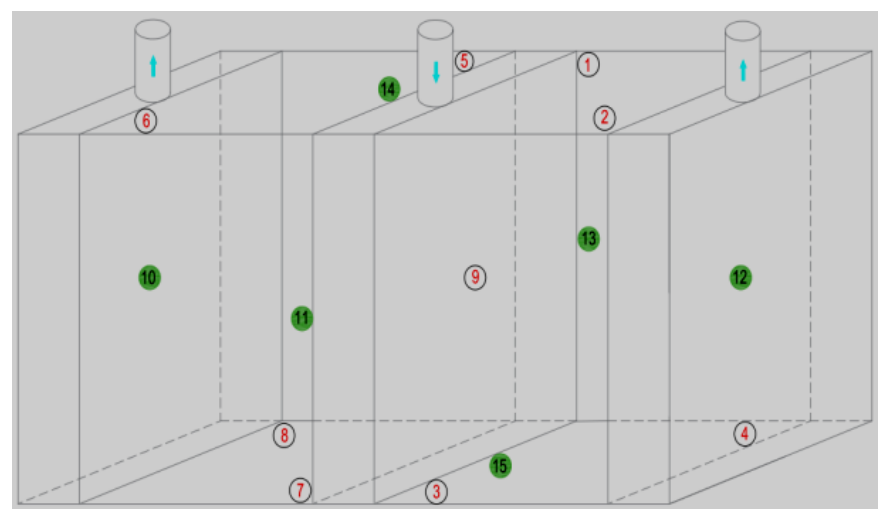

Figure 2: place the temperature probes for the volumes greater than $2 \mathrm{~m}^{3}$ and less than or equal to $20 \mathrm{~m}^{3}$. 
Set the characterization time at 60 minutes at a frequency of 1 reading every minute after stabilizing the temperature in the chamber.

Also note the values indicated by the display of the chamber at the beginning, middle and end of characterization on a table then calculate:

- The average temperature by probes $\mathrm{X}_{\mathrm{mj}}$;

- The air temperature $X_{\text {air }}$;

- Homogeneity of temperature. $\mathrm{GX}_{\mathrm{E}}$ :

$$
\mathbf{G X} E=\operatorname{Max} \text { value }(\mathbf{X} m j+\mathbf{U} m j)-\mathbf{M i n} \text { value }(\mathbf{X} m j-\mathbf{U} m j)
$$

- Maximum homogeneity $\mathrm{GX}_{\mathrm{M}}$ :

$$
\mathbf{G X} M=\text { Max value }(\mathbf{X} m j+\mathbf{U} j)-\text { Min value }(\mathbf{X} m j-\mathbf{U} j)
$$

The verification of the homogeneity of the temperature is considered to be compliant if the interval $\left(X_{m j}-U_{m j}\right.$; $\left.\mathrm{X}_{\mathrm{mj}}+\mathrm{U}_{\mathrm{mj}}\right)$ is included in the MPE interval.

The Maximum Permissible Error (MPE) is: $40,0 \pm 3,0^{\circ} \mathrm{C}$. Here an example:

- $\quad \mathbf{X}_{\mathrm{air}}=39,83^{\circ} \mathrm{C}$.

- $\mathbf{G X}_{\mathrm{E}}=40,49-39,30=1,19^{\circ} \mathrm{C} \leq 3,0^{\circ} \mathrm{C}$.

- $\mathbf{G X}_{\mathrm{M}}=40,55-39,10=1,45^{\circ} \mathrm{C} \leq 3,0^{\circ} \mathrm{C}$.

\section{3-4-2 Verification of the stability of the temperature at $40^{\circ} \mathrm{C}$ "Empty chamber".}

Calculate:

- Stability at each recording point $\mathrm{SX}_{\mathrm{j}}$,

- Maximum stability $\mathrm{SX}_{\mathrm{M}}$,

The values of stability are calculated as a guide to know the amplitude of the regulation. There are no compliance standards. If the stability values do not exceed $6^{\circ} \mathrm{C}$, this reflects good regulation.

\section{3-4-3 Determination of the temperature setpoint deviation at $40^{\circ} \mathrm{C}$.}

The setpoint deviation is the difference between the setpoint value Xco and the average value measured in the air of the Xair chamber.

$$
\Delta \text { Xco }=\text { Xco }- \text { Xair }
$$

Acceptance criteria: The absolute value of the difference between the setpoint temperature and the air temperature must be less than or equal to the extended uncertainty.

Example:

$$
\mid \mathbf{X c o} \text { - Xair } \mid \leq \mathbf{U}
$$

Table 4: the data of setpoint devation of the temperature at $40^{\circ} \mathrm{C}$.

\begin{tabular}{|c|c|}
\hline $\mathbf{X}_{\text {air }}\left({ }^{\circ} \mathrm{C}\right)$ & 39,83 \\
\hline $\mathbf{X}_{\mathbf{c o}}\left({ }^{\circ} \mathrm{C}\right)$ & 40,00 \\
\hline $\mathbf{U}\left({ }^{\circ} \mathrm{C}\right)$ & 0,22 \\
\hline $\mathbf{X}_{\mathbf{c o}}-\mathbf{X}_{\text {air }}\left({ }^{\circ} \mathrm{C}\right)$ & 0,17 \\
\hline Standard & $\left|\mathbf{X}_{\mathbf{c o}}-\mathbf{X}_{\text {air }}\right| \leq \mathbf{U}$ \\
\hline Statut $(\mathrm{C} / \mathrm{NC})$ & Compliant \\
\hline
\end{tabular}




\section{3-4-4 Determination of the error indication of the temperature at $40^{\circ} \mathrm{C}$ :}

To do this: Note the values indicated on the display of the chamber at the beginning, middle and in the end of characterization. The indication error is the difference between the average value of indication $X_{\text {in }}$ (average of the three values: Beginning - middle - end) and the average value measured in the air of the Xair chamber. Indicator deviation:

$$
\Delta \text { Xin }=\text { Xin }- \text { Xair (5) }
$$

The absolute value of the difference between the air temperature and the temperature indicated by the chamber shall be less than or equal to the extended uncertainty $U$ given in the equation (4).

Table 5: The error indication data of the temperature at $40^{\circ} \mathrm{C}$.

\begin{tabular}{|c|c|c|c|c|}
\hline \multicolumn{4}{|c|}{ Xair $\left({ }^{\circ} \mathrm{C}\right)$} & 39,83 \\
\hline \multirow{2}{*}{ Xind $\left({ }^{\circ} \mathrm{C}\right)$} & Beginning & Middle & End & \multirow{2}{*}{40,15} \\
\hline & $39.9^{\circ} \mathrm{C}$ & $40,0^{\circ} \mathrm{C}$ & $40,0^{\circ} \mathrm{C}$ & \\
\hline \multicolumn{4}{|c|}{$\mathbf{U}\left({ }^{\circ} \mathrm{C}\right)$} & 0,22 \\
\hline \multicolumn{4}{|c|}{$\mathbf{X}_{\text {ind }}-\mathbf{X}_{\text {air }}\left({ }^{\circ} \mathrm{C}\right)$} & 0,17 \\
\hline \multicolumn{4}{|c|}{ Standard } & $\left|\mathbf{X}_{\text {ind }}-\mathbf{X}_{\text {air }}\right| \leq \mathbf{U}$ \\
\hline \multicolumn{4}{|c|}{ Statut (C/NC) } & Compliant \\
\hline
\end{tabular}

\section{3-4-5 Temperature recovery time at $40^{\circ} \mathrm{C}, 50^{\circ} \mathrm{C}$ and $60^{\circ} \mathrm{C}$ :}

Principle: Since the chamber is in established regime, the recovery time is the time required for the environment to regain the established regime after a disturbance. This time is measured from a voluntary action (door opening, power cut, etc.). In the case of a door opening, the opening time is one minute with a total opening.
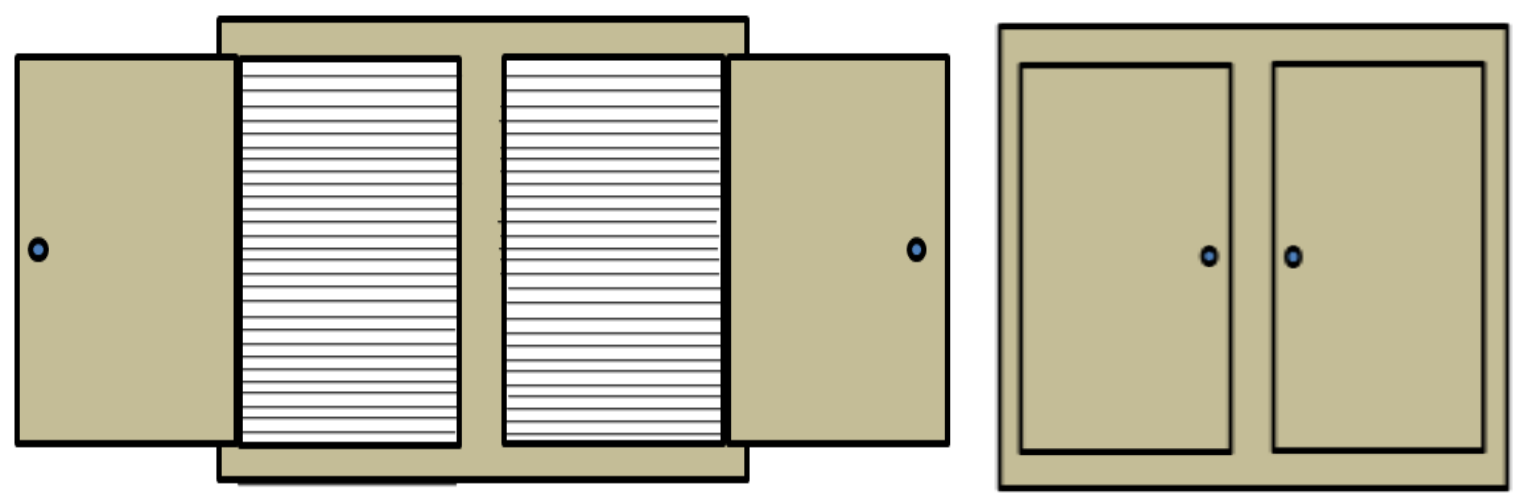

Figure 3: Opening and closing door of the chamber.

The Time is counted from the end of the voluntary disturbance. Recovery time is expressed in minutes. Exampale:

- Recovery time Equal 1 minute 02 seconds for temperature $40^{\circ} \mathrm{C}$.

- Recovery time Equal 1 minute 22 seconds for temperature $50^{\circ} \mathrm{C}$.

- Recovery time Equal 1 minute 53 seconds for temperature $60^{\circ} \mathrm{C}$

This is a very important factor in the characterization of climatic and thermostatic chambers. This makes it possible to gain a visibility about the time required for the regime to be established after a disturbance (power failure, opening of doors during cycles). 


\section{3-4 Compliance and Judgment Rules:}

In the absence of specific rules of conformity defined by the customer and formalized at the time of the request, the conformity of an chamber shall be established in accordance with the following rules:

\section{3-4-1 The compliant rule is defined in the standard NF X 15-140 :}

The chamber is declared compliant if, for each specified parameter, the average of all measurements Xmj of each probe and its associated extended uncertainty Umj belongs to the range of MPE located around the specified value:

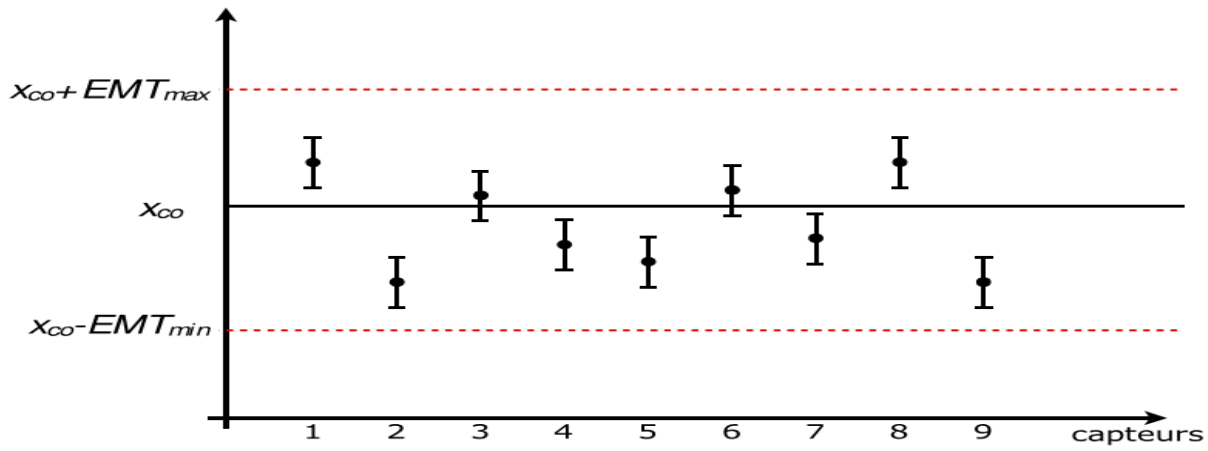

Figure 4: Example of compliant chamber according to NF X 15-140.

The deviation setpoint $\Delta \mathrm{X}_{\mathrm{co}}$ found between the Xair value of a parameter of the chamber and the desired condition $X_{\text {co }}$ of this parameter is taken into account when it is significant, in particular if $\left|X_{\text {air }}-X_{c o}\right|>U$.

\section{3-4-2 Verification according to standard NF EN 60068-3:}

The compliance rule is not formally defined in standard NF EN 60068-3-5. The interpretation that follows from the elements of the standard is as follows: the chamber is declared compliant if the average value of the Xair parameter, increased or decreased by the associated uncertainty air $U\left(X_{\text {air }}\right)$, belongs to the maximum error range Tolerated.

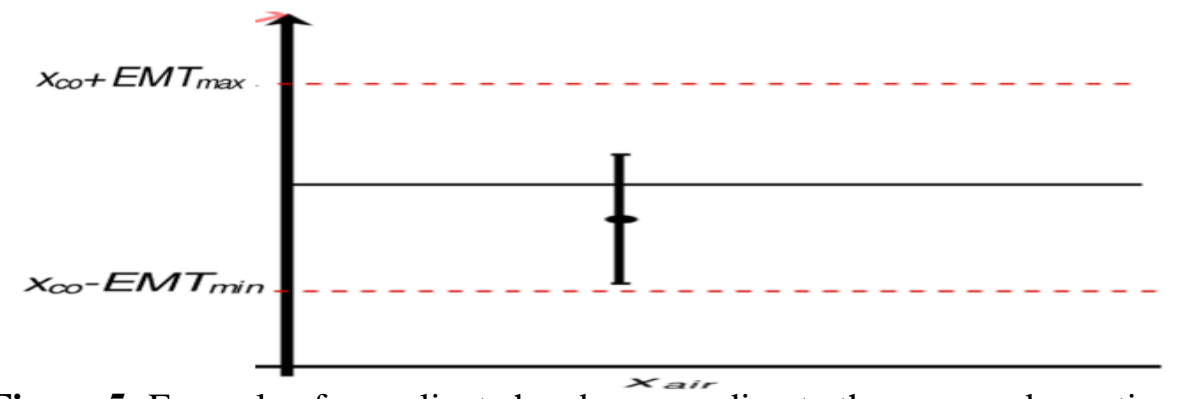

Figure 5: Example of compliant chamber according to the proposed equation for standard NF EN 60068-3-5 [2].

\section{Conclusion:}

The qualification of climatic and thermostatic chambers is not based on a single standard, but it also requires the control of several standards such as NF X 15-140, NF EN 60068-3 and the requirements of the Good Manufacturing Practices (GMP). So, currently the qualifiers only take into account the standard NF X 15-140 or NF EN 60068-3 to make their qualification, without giving importance to other criteria that must be treated or accurately tested and that are not mentioned in these standards, but they are cited in other references such as GMP (Good Manufacturing Practices). Because with the standards NF X 15-140 or NF EN 60068-3 we can only judge on the conformity of the distribution of temperature and humidity into the chamber. That is to say, in qualification terms, it is the last step of the qualification, which is the PQ (Performance Qualification). While in the GMP, we find the other steps of qualification, which are the IQ (Installation Qualification) and the OQ (Operational Qualification) and even in the GMP, they are not well detailed. Thanks to the use of the characterization standards such as NF X 15-140 and NF EN 60068-3 and the requirements of the Good 
Manufacturing Practices, we have been able to develop a standard qualification protocol. Indeed, this protocol could be used for all climatic and thermostatic chambers for the purpose of their qualification. Also, it could allow us to make a judgment on the conformity or not of the chamber to qualify.

\section{References}

[1] NF X 15-140 Mesure de l'humidité de l'air - Enceintes climatique et thermostatique - Caractérisation et vérification, Mai 2013.

[2] NF EN 60068-3-5, Essais d'environnement-Partie 3-5: documentation d'accompagnement et guide Confirmation des performances des chambres d'essais en température, Aout 2002.

[3] Good Manufacturing Practices (GMP) N 12 bis and annexe 12015 ).

[4] Claire EMAILLE : Thèse pour le diplôme d'état de docteur en pharmacie «Qualification d'une ligne de conditionnement » le 28 Mars 2003, Léon France.

[5] Nicholas BOUILLON, Laure DOMENECH, Boris GEYNET et David BENHAMOU Caractérisation et vérification des enceintes $\mathrm{N}^{\circ} 27$, Février 2011. 